\title{
Implementation and training with laparoscopic distal pancreatectomy: 23-year experience from a high-volume center
}

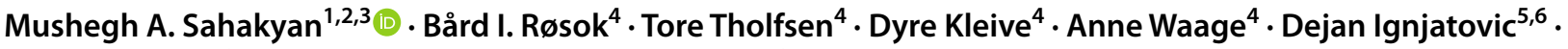 \\ Trond Buanes ${ }^{4,6} \cdot$ Knut Jørgen Labori ${ }^{4} \cdot$ Bjørn Edwin ${ }^{1,4,6}$
}

Received: 6 October 2020 / Accepted: 9 January 2021 / Published online: 3 February 2021

(c) The Author(s) 2021

\begin{abstract}
Background Distal pancreatectomy is the most common procedure in minimally-invasive pancreatic surgery. Data in the literature suggest that the learning curve flattens after performing up to 30 procedures. However, the exact number remains unclear.

Methods The implementation and training with laparoscopic distal pancreatectomy (LDP) in a high-volume center were studied between 1997 and 2020. Perioperative outcomes and factors related to conversion were assessed. The individual experiences of four different surgeons (pioneer and adopters) performing LDP on a regular basis were examined.

Results Six hundred forty LDPs were done accounting for $95 \%$ of all distal pancreatectomies performed throughout the study period. Conversion was needed in 14 (2.2\%) patients due to intraoperative bleeding or tumor adherence to the major vasculature. Overall morbidity and mortality rates were 35 and $0.6 \%$, respectively. Intra- and postoperative outcomes did not change for any of the surgeons within their first 40 cases. Operative time significantly decreased after the first 80 cases for the pioneer surgeon and did not change afterwards although the proportion of ductal adenocarcinoma increased. Tumor size increased after the first 80 cases for the first adopter without affecting the operative time.

Conclusions In this nearly unselected cohort, no significant changes in surgical outcomes were observed throughout the first 40 LDPs for different surgeons. The exact number of procedures required to overcome the learning curve is difficult to determine as it seems to depend on patient selection policy and specifics of surgical training at the corresponding center.
\end{abstract}

Keywords Laparoscopy $\cdot$ Pancreatectomy $\cdot$ Conversion $\cdot$ Morbidity $\cdot$ Adenocarcinoma

The proportion of minimally-invasive pancreatic resections performed worldwide has been steadily increasing

Mushegh A. Sahakyan

sahakyan.mushegh@gmail.com

1 The Intervention Centre, Rikshospitalet, Oslo University Hospital, Oslo, Norway

2 Department of Research \& Development, Division of Emergencies and Critical Care, Oslo University Hospital, Oslo, Norway

3 Department of Surgery N1, Yerevan State Medical University After M. Heratsi, Yerevan, Armenia

4 Department of Hepato-Pancreato-Biliary Sugery, Rikshospitalet, Oslo University Hospital, Oslo, Norway

5 Department of Digestive Surgery, Akershus University Hospital, Lørenskog, Norway

6 Institute of Clinical Medicine, Faculty of Medicine, University of Oslo, Oslo, Norway
$[1,2]$. Distal pancreatectomy is the most common procedure among those [3, 4]. Randomized controlled trials have shown clear advantages for minimally-invasive distal pancreatectomy (MIDP) over its open counterpart [5, 6]. As a result, current international guidelines recommend considering MIDP as a primary approach in patients with benign lesions and low-grade malignancies in the body and tail of the pancreas $[2,7,8]$.

According to the literature, up to 30 procedures should be performed to overcome the learning curve and reach proficiency in MIDP [9-15]. However, interpretation of the literature data is difficult due to several reasons. First, findings from different studies are hardly comparable as they are based on both single- and multicenter experiences, as well as on single-surgeon series. Second, these reports often include a relatively small number of highly selected patients. Finally, analysis of pooled data from multiple surgeons with different 
levels of expertise in MIDP complicates the understanding of its true learning curve.

The aim of our report was to analyze 23-year experience with laparoscopic distal pancreatectomy (LDP) in a highvolume center for pancreatic surgery. The focus was on the implementation and training with this procedure for different surgeons.

\section{Materials and methods}

\section{Study design}

Study included a nearly unselected cohort of patients who underwent LDP at Oslo University Hospital, Rikshospitalet from April 1997 to June 2020. All patients were evaluated at the multidisciplinary team meeting prior to surgery and the final choices of management were made. The international guidelines were followed when deciding on the inclusion/ exclusion criteria for surgery. Pancreatic cancer itself has never been an exclusion criteria. No preoperative histology was performed in these patients, i.e. those with radiological suspicion of cancer were considered for surgery. Since the pioneer surgeon performed the first LDP at our institution in April 1997, it has become a standard procedure for patients with lesions in the body and tail of the pancreas. Open distal pancreatectomy was reserved primarily for the patients with vascular involvement, where major vascular resection and reconstruction were needed. As a result, it was applied in only $5 \%$ of patients throughout the study period (Supplementary Fig. 1).

Four different surgeons performed the evaluated procedures. The pioneer surgeon performed his initial procedures in 1997 at the time of the advent of laparoscopic pancreatic surgery without receiving specific training or supervision. The first adopter was trained by the pioneer surgeon in mid2000s and became an expert over time. During the last four years two trainees were trained by the pioneer and expert to be able to perform LDP on a regular basis. The expert and trainees all had broad experience in performing laparoscopic cholecystectomy, appendectomy, colon resection or gastric bypass/gastroenterostomy when initiating their learning curve in LDP. All three trainees received a multimodal surgical education including training during their fellowship in open pancreatic surgery, acting as the camera holder first during LDP and then performing LDP. The above-mentioned four surgeons have done the largest number of LDPs at our institution accounting for $87.5 \%$ of these procedures performed throughout the study period. The extent of LDP and its technique have not significantly changed over time and the principles introduced by the pioneer surgeon have been followed by the adopters.
Information on patient demographics, comorbidities, and clinical characteristics, history of previous upper abdominal surgery, intraoperative parameters and postoperative results was retrieved from a prospectively maintained database. The experience with LDP was assessed. Procedures that were eventually converted to open surgery were also studied to identify specific factors leading conversion. Potential changes in perioperative results of LDP associated with an individual experience of the operating surgeon were assessed for the above-mentioned surgeons. The study was approved by the hospital review board according to the guidelines provided by the regional ethics committee.

The first 40 procedures of each surgeon were selected to examine the initial experience with LDP. Perioperative outcomes in these 40 cases were examined in 4 groups consisting of 10 patients each. The results of the first 40 LDPs were compared with the subsequent experience with this procedure ( 40 by 40 ). For better understanding of the individual results of each surgeon, the outcomes of the first 30 cases operated by the adopters without senior surgeon supervision/ assistance were compared to those of a pioneer surgeon.

\section{Definitions}

Previous upper abdominal surgery was defined as a surgical procedure performed in the upper portion of the peritoneal cavity, i.e. involving any organ located higher than the umbilicus. These included procedures on the hepato-pancreato-biliary system, stomach, spleen, small intestine, kidney, adrenal glands, upper retroperitoneum, and diaphragm. Colectomy involving the upper abdomen in the dissection area was considered as previous upper abdominal surgery.

Multivisceral resections included extended distal pancreatectomy and non-contiguous organ resection in the setting of distal pancreatectomy, as defined by the International Study Group for Pancreatic Surgery (ISGPS) [16]. Conversion was defined as laparotomy during LDP not related to the specimen extraction. Oslo classification based on Satava approach to surgical error evaluation was used to define and grade intraoperative adverse incidents $[17,18]$. Postoperative morbidity was defined based on the Accordion Severity Grading System [19]. Grade $\geq$ III complications were considered severe. Postoperative pancreatic fistula (POPF) was reported according to the 2016 update from the ISGPS [20]. Postpancreatectomy hemorrhage was defined and classified as suggested by the ISGPS [21]. The 90 days from surgery definition was used for mortality and readmission [22].

\section{Technique}

Patients are placed on a modified supine position with the left side raised $30^{\circ}-45^{\circ}[23,24]$. The first $12-\mathrm{mm}$ trocar is placed through the umbilicus. Then 5-mm trocar is placed in 
the midline between the xiphoid process and the umbilicus. The third is 12-mm trocar (camera) - lateral to the left rectus muscle at the level of the umbilicus. Finally, one 5-mm trocar is placed in the left subcostal region, on the midclavicular line. For the centrally located pancreatic tumors the second trocar could be placed laterally from the right rectus muscle and above the umbilicus.

The procedure usually starts with the mobilization of splenic flexure of the colon followed by division of the short gastric vessels. However, the short gastric vessels are preserved if spleen-preserving LDP is performed. After releasing the transverse colon medially, the inferior margin of the pancreas is dissected free from the retroperitoneum. After the mobilization of the pancreatic body and tail is completed, and the splenic vessels are identified. In case of PDAC, the dissection plane is normally extended to the origin of the splenic artery and to the confluence of the splenic and superior mesenteric veins. Laparoscopic intraoperative ultrasound is often used to identify major vascular structures and examine their relationship with the lesion. The splenic vessels are divided by an Endo-GIA stapling device (Medtronic, Minneapolis, MA, USA). Regional lymph nodes (around the celiac trunk, along the splenic artery/inferior border of the pancreas and in the splenic hilum) are removed en bloc with the specimen. The level of pancreatic gland division depends on tumor location. Pancreatic body or neck is transected using a $60 \mathrm{~mm}$ EndoGIA stapling device (Medtronic, Minneapolis, MA, USA). After full mobilization of the spleen, the specimen is placed into the EndoCatch (Medtronic, Minneapolis, MA, USA) and retrieved through a small extension of the umbilical port incision. The use of the fibrinogen/thrombin-coated collagen sponge or glue on the pancreatic stump is left at surgeon's discretion. One drain is routinely placed near the pancreatic stump.

\section{Statistics}

The continuous data were expressed as mean $( \pm$ standard deviation) or median (range) depending on data distribution. The analysis of variance (ANOVA) was used to compare normally distributed continuous data, and the post hoc test was used to verify statistically significant differences between the means. The Kruskal-Wallis and two-sided Mann-Whitney U-test were used for not normally distributed continuous data. The categorical variables were expressed as numbers (percentages). To compare these, the Chi-square test or Fisher's exact test were. The two-sided $p$-value $<0.05$ was considered statistically significant.

\section{Results}

\section{Overview}

A total number of 640 patients underwent LDP throughout the study period. Ductal adenocarcinoma was the indication for surgery in $138(21.6 \%)$ patients (Table 1). The pioneer, expert, trainee 1 and trainee 2 performed 304 $(47.5 \%), 169(26.4 \%), 39(6.1 \%)$ and $48(7.5 \%)$ procedures, respectively. The remaining $80(12.5 \%)$ patients were operated by other surgeons, mostly under the supervision of the pioneer or expert. Median operative time and blood loss were $160 \mathrm{~min}$ and $60 \mathrm{ml}$, respectively. Conversion to open surgery was needed in $14(2.2 \%)$ patients. Postoperative complications developed in 224 (35\%) patients including 119 (18.6\%) with grade B/C pancreatic fistula. Reoperation was done in 34 (5.3\%) cases, and median postoperative length of stay was 5 days.

\section{Conversion}

Detailed description of cases that required conversion to open surgery is given in Table 2 . The majority of those (10 of 14) were operated for pancreatic cancer. In 9 cases, the operating surgeon had individual record of more than 40 LDPs performed. The reasons for conversion were adherence to the major vessels potentially requiring vascular resection and reconstruction $(n=8)$ and major intraoperative bleeding $(n=6)$. Major vascular resection with reconstruction was performed in 7 cases and multivisceral resection without vascular reconstruction was done in 3 patients. Eight patients developed postoperative complications.

\section{Implementation and training}

The individual experience with the first 40 LDPs was analyzed for the pioneer surgeon (Table $3 \mathrm{a}$ ). The body mass index of the first 10 patients was significantly higher compared to the rest ( 30.6 vs 24.6 vs 23.6 vs $24.6 \mathrm{~kg} / \mathrm{m}^{2}$, $p=0.03$ ), and the tumor size was significantly larger in the last 10 patients ( 23.5 vs 25 vs 19.5 vs $44.5 \mathrm{~mm}, p=0.03$ ). No statistically significant changes were detected in other perioperative parameters including operative time, blood loss, conversion, and complications. The results of the 40 cases operated by the pioneer surgeon were compared with the next 6 consecutive periods including 40 cases each (Table 3b). A significant decrease in median operative time was detected after the first 80 cases. Thereafter, it did not change. The proportion of PDAC significantly increased, and the number of spleen-preserving LDP decreased in the 
Table 1 Experience with laparoscopic distal pancreatectomy at Oslo University Hospital, Rikshospitalet: 1997-2020

\begin{tabular}{|c|c|}
\hline Parameters & $n=640$ \\
\hline Age, years, mean (SD) & $61(14)$ \\
\hline Body mass index, $\mathrm{kg} / \mathrm{m}^{2}$, mean (SD) & $25.8(4.6)$ \\
\hline \multicolumn{2}{|l|}{ Gender, $n(\%)$} \\
\hline Female/male & $331(51.7 \%) / 309(48.3 \%)$ \\
\hline Cardiovascular diseases, $n(\%)$ & $110(17.4 \%)$ \\
\hline Chronic obstructive pulmonary disease, $n(\%)$ & $102(15.9 \%)$ \\
\hline Diabetes mellitus, $n(\%)$ & $102(15.9 \%)$ \\
\hline Total number of comorbidities, mean (SD) & $1.4(1.3)$ \\
\hline ASA score $\geq \mathrm{III}, n(\%)$ & $203(32 \%)$ \\
\hline Diagnosis of PDAC, $n(\%)$ & $138(21.6 \%)$ \\
\hline Tumor size, mm, median (range) & $34(4-180)$ \\
\hline \multicolumn{2}{|l|}{ Surgeons, $n(\%)$} \\
\hline Pioneer & $304(47.5 \%)$ \\
\hline Expert & $169(26.4 \%)$ \\
\hline Trainee 1 & $39(6.1 \%)$ \\
\hline Trainee 2 & $48(7.5 \%)$ \\
\hline Other & $80(12.5 \%)$ \\
\hline Spleen-preserving procedure, $n(\%)$ & $91(14.2 \%)$ \\
\hline Multivisceral resection, $n(\%)$ & $87(13.6 \%)$ \\
\hline Conversion, $n(\%)$ & $14(2.2 \%)$ \\
\hline Operative time, min, median (range) & $160(30-560)$ \\
\hline Estimated blood loss, ml, median (range) & $60(10-6250)$ \\
\hline Perioperative red blood cell transfusion, $n(\%)$ & $57(9 \%)$ \\
\hline Postoperative complications, $n(\%)$ & $224(35 \%)$ \\
\hline Severe complications (grade $\geq \mathrm{III}), n(\%)$ & $139(21.7 \%)$ \\
\hline Clinically relevant pancreatic fistula, $n(\%)$ & $119(18.6 \%)$ \\
\hline Grade $\mathrm{B} / \mathrm{C}$ hemorrhage, $n(\%)$ & $33(5.2 \%)$ \\
\hline Reoperation, $n(\%)$ & $34(5.3 \%)$ \\
\hline Readmission, $n(\%)$ & $62(9.7 \%)$ \\
\hline 90-day mortality, $n(\%)$ & $4(0.6 \%)$ \\
\hline Postoperative length of stay, days, median (range) & $5(2-81)$ \\
\hline
\end{tabular}

ASA American Society of Anesthesiologists, $P D A C$ pancreatic ductal adenocarcinoma later periods. Other intra- and postoperative parameters remained similar.

The expert surgeon performed the first LDP after having assisted the pioneer on 9 LDPs. The experience with first 40 cases was analyzed (Table 3c). No significant changes in perioperative outcomes were observed although the number of cases assisted by the pioneer surgeon significantly decreased ( 10 vs 3 vs 2 vs $1, p<0.01)$. No conversions were performed in this period. The analysis of the next 3 consecutive periods including 40 procedures each demonstrated significant increase in tumor size following the first 80 procedures (Table $3 \mathrm{~d}$ ). The proportion of spleen-preserving procedures decreased after period 1. Operative time did not change over time. The number of cases assisted/supervised by the pioneer surgeon decreased from $40 \%$ in the period 1 to $2.5 \%, 5 \%$ and $0 \%$ in the periods 2, 3 and 4 , respectively $(p<0.01)$. Intra- and postoperative outcomes were comparable.

Before performing their first LDP trainees 1 and 2 had assisted on 11 and 7 procedures, respectively. Shorter time span was required for the trainees to perform their first 30-40 LDP compared with the pioneer and the expert (Supplementary Fig. 2). Their initial experience with LDP is presented in Table $4 \mathrm{a}$ and $\mathrm{b}$. The number of cases assisted/ supervised by either the pioneer or expert surgeons significantly decreased over time. Operative time and other perioperative outcomes did not change.

The individual experiences of the adopters (expert and trainees) with their first 30 non-supervised LDPs were compared to the outcomes of the first 30 consecutive LDPs performed by the pioneer surgeon (Table 5). To reach a total number of 30 non-supervised LDPs, 46, 39 and 48 cases 
Table 2 Description of cases that required conversion from laparoscopic to open distal pancreatectomy

\begin{tabular}{|c|c|c|c|c|c|c|c|}
\hline No & $\begin{array}{l}\text { Patient } \\
\text { BMI }(\mathrm{kg} / \\
\left.\mathrm{m}^{2}\right)\end{array}$ & Diagnosis & $\begin{array}{l}\text { Tumor } \\
\text { size } \\
(\mathrm{mm})\end{array}$ & $\begin{array}{l}\text { Sur- } \\
\text { geon's } \\
\text { case no }\end{array}$ & Reason of conversion & Open procedure & $\begin{array}{l}\text { Postoperative Complica- } \\
\text { tions }\end{array}$ \\
\hline 1 & 25.2 & Chronic pancreatitis & - & 3 & Intraoperative bleeding & DP & - \\
\hline 2 & 26.4 & Serosal cystadenoma & 60 & 43 & $\begin{array}{l}\text { Bleeding from the upper } \\
\text { pole of the spleen }\end{array}$ & $\begin{array}{l}\text { DP; left adrenalectomy; } \\
\text { stomach resection }\end{array}$ & - \\
\hline 3 & 29.4 & NEN & 55 & 51 & $\begin{array}{l}\text { Bleeding from the } \\
\text { splenic vein }\end{array}$ & $\begin{array}{l}\text { DP; left adrenalectomy; } \\
\text { stomach resection }\end{array}$ & Thrombosis of the SMV \\
\hline 4 & 25.8 & PDAC & 17 & 43 & $\begin{array}{l}\text { Tumor adherence to the } \\
\text { SMV }\end{array}$ & $\begin{array}{l}\text { DP; resection of the } \\
\text { SMV }\end{array}$ & - \\
\hline 5 & 25.9 & PDAC & 88 & 44 & $\begin{array}{l}\text { Tumor adherence to the } \\
\text { portal vein }\end{array}$ & $\begin{array}{l}\text { DP; resection of the por- } \\
\text { tal vein; liver resection }\end{array}$ & - \\
\hline 6 & 25.4 & $\begin{array}{l}\text { Adenosquamous carci- } \\
\text { noma }\end{array}$ & 85 & 54 & $\begin{array}{l}\text { Bleeding from the portal } \\
\text { vein }\end{array}$ & $\begin{array}{l}\text { DP; resection of the } \\
\text { portal vein }\end{array}$ & PF; hemorrhage \\
\hline 7 & 24.4 & PDAC & 35 & 57 & $\begin{array}{l}\text { Tumor infiltration into } \\
\text { the duodenum and } \\
\text { adherence to the mid- } \\
\text { dle colic artery }\end{array}$ & $\begin{array}{l}\text { DP; resection of the } \\
\text { duodenum }\end{array}$ & $\mathrm{PF}$ \\
\hline 8 & 14.9 & PDAC & 55 & 59 & $\begin{array}{l}\text { Bleeding from the } \\
\text { splenic vein }\end{array}$ & DP & - \\
\hline 9 & 35.2 & PDAC & 44 & 110 & $\begin{array}{l}\text { Bleeding from the coe- } \\
\text { liac trunk }\end{array}$ & $\begin{array}{l}\text { DP; repair of the vascu- } \\
\text { lar injury }\end{array}$ & Pulmonary embolism; PF \\
\hline 10 & 26.2 & PDAC & 77 & 129 & $\begin{array}{l}\text { Tumor adherence to the } \\
\text { portovenous conflu- } \\
\text { ence }\end{array}$ & $\begin{array}{l}\text { DP; resection of the } \\
\text { portal vein }\end{array}$ & Urinary incontinence \\
\hline 11 & 26.1 & $\begin{array}{l}\text { Adenosquamous carci- } \\
\text { noma }\end{array}$ & 70 & 24 & $\begin{array}{l}\text { Tumor adherence to the } \\
\text { portal vein }\end{array}$ & $\begin{array}{l}\text { DP; resection of the } \\
\text { portal vein }\end{array}$ & - \\
\hline 12 & 38.9 & Serosal cystadenoma & 45 & 12 & $\begin{array}{l}\text { Tumor adherence to the } \\
\text { SMV }\end{array}$ & $\begin{array}{l}\text { DP; resection of the } \\
\text { SMV }\end{array}$ & $\begin{array}{l}\text { Hemorrhage; thrombosis } \\
\text { of the SMV; PF }\end{array}$ \\
\hline 13 & 28.1 & PDAC & 9 & 19 & $\begin{array}{l}\text { Fibrosis in the surgi- } \\
\text { cal area, suspicion of } \\
\text { vascular affection }\end{array}$ & DP & $\mathrm{PF}$ \\
\hline 14 & 33.2 & PDAC & 44 & 36 & $\begin{array}{l}\text { Suspicion of vascular } \\
\text { affection }\end{array}$ & DP & Pulmonary embolism \\
\hline
\end{tabular}

$D P$ distal pancreato-splenctomy, $N E N$ neudoendocrine neoplasia, $S M V$ superior mesenteric vein, $P D A C$ pancreatic ductal adenocarcinoma, $P F$ pancreatic fistula

were required for the expert, trainee 1 and 2 , respectively. These included procedures assisted or supervised by a senior surgeon.

Preoperative parameters such as patient demographics, body mass index, history of previous upper abdominal surgery, tumor size and diagnosis of PDAC was similar for all surgeons. The rate of spleen preserving procedures was significantly higher for the pioneer and expert compared to the trainees -23.3 vs 36.7 vs 3.3 vs $3.3 \%$, $(p=0.001)$, respectively. The proportion of multivisceral resections was significantly higher in expert's experience compared with the trainees -20 vs 3.3 vs $0 \%(p=0.015)$, respectively. Median operative time significantly decreased when comparing the outcomes of the pioneer and adopters - 238 vs 188 vs 161 vs $156 \min (p<0.001)$. The incidences of intraoperative adverse events and conversion were similar. Postoperative outcomes including complications, pancreatic fistula, hemorrhage, reoperation and readmission were comparable. None of the patients died within 90 days of surgery. Median length of postoperative stay was significantly shorter for the trainee 2 compared with the pioneer and expert (4 vs 6 vs 6 $(p=0.02)$, respectively).

\section{Discussion}

Our experience with LDP is based on its implementation and standardization of intraoperative steps by a pioneer surgeon followed by the stepwise training for the adopters. The consecutive phases of training included assisting the pioneer surgeon (and learning the intraoperative steps) (i), performing surgery with (ii) and without supervision (iii). As a result, satisfactory results were registered for the adopters. Interestingly, the conversion rate in this series was 
Table 3 Experiences of the pioneer and expert surgeons with LDP: first 40 cases of the pioneer (a); first and subsequent 40 cases of the pioneer (b); first 40 cases of the expert (c); first and subsequent 40 cases of the expert (d)

\begin{tabular}{|c|c|c|c|c|c|c|c|c|}
\hline \multicolumn{2}{|l|}{$\begin{array}{l}\text { Parameters } \\
\text { (a) }\end{array}$} & \multicolumn{2}{|l|}{$1-10$} & $11-20$ & \multicolumn{2}{|l|}{$21-30$} & $31-40$ & $p$-value \\
\hline \multicolumn{2}{|l|}{ Age, years, mean (SD) } & \multicolumn{2}{|l|}{$56.7(12.2)$} & $56(15.2)$ & \multicolumn{2}{|l|}{$62.3(10)$} & $59.8(12.9)$ & 0.41 \\
\hline \multicolumn{2}{|c|}{$\mathrm{BMI}, \mathrm{kg} / \mathrm{m}^{2}$, mean $(\mathrm{SD})^{\mathrm{a}}$} & \multicolumn{2}{|l|}{$30.6(7.7)$} & $24.6(1.9)$ & \multicolumn{2}{|l|}{$23.6(3.2)$} & $24.6(3.3)$ & 0.03 \\
\hline \multicolumn{2}{|l|}{ PUAS, $n$} & \multicolumn{2}{|l|}{4} & 6 & \multicolumn{2}{|l|}{6} & 3 & 0.54 \\
\hline \multicolumn{2}{|l|}{ Diagnosis (PDAC), $n$} & \multicolumn{2}{|l|}{1} & 2 & \multicolumn{2}{|l|}{2} & 2 & 1.0 \\
\hline \multicolumn{2}{|c|}{ Tumor size, mm, median (range) ${ }^{b}$} & \multicolumn{2}{|c|}{$23.5(4-70)$} & $(11-75)$ & $19.5(15-2$ & 44.5 & (29-70) & 0.03 \\
\hline Spleen-preserving pro & ocedure, $n$ & 1 & 2 & & 4 & 1 & & 0.46 \\
\hline Multivisceral resectio & n, $n$ & 0 & 2 & & 2 & 1 & & 0.73 \\
\hline Blood loss, ml, media & an (range) & $100(30-10$ & 000) & $0(30-1500)$ & $80(30-350$ & 250 & $(30-3000)$ & 0.5 \\
\hline Operative time, min, $\mathrm{n}$ & median (range) & $248(125-$ & 360) & $3(180-520)$ & $215(123-3$ & 188 & $(135-260)$ & 0.16 \\
\hline Intraoperative adverse & events, $n$ & 2 & 3 & & 0 & 2 & & 0.46 \\
\hline Conversion, $n$ & & 1 & 0 & & 0 & 0 & & 1.0 \\
\hline Postoperative complic & cations, $n$ & 3 & 6 & & 4 & 4 & & 0.68 \\
\hline Severe complications, & & 3 & 3 & & 2 & 2 & & 1.0 \\
\hline CR-PF, $n$ & & 2 & 2 & & 0 & 1 & & 0.73 \\
\hline Grade $\mathrm{B} / \mathrm{C}$ hemorrhag & ge, $n$ & 1 & 1 & & 0 & 1 & & 1.0 \\
\hline Reoperation, $n$ & & 0 & 0 & & 0 & 1 & & 1.0 \\
\hline Mortality, $n$ & & 0 & 0 & & 0 & 0 & & - \\
\hline Hospital stay, days, m & ledian (range) & $6(4-8)$ & & 2-12) & $6(2-11)$ & $5(3-$ & $-16)$ & 0.73 \\
\hline Parameters & $\begin{array}{l}\text { Period } 1 \\
(1-40)\end{array}$ & $\begin{array}{l}\text { Period } 2 \\
(41-80)\end{array}$ & $\begin{array}{l}\text { Period } 3 \\
(81-120)\end{array}$ & $\begin{array}{l}\text { Period } 4 \\
(121-160)\end{array}$ & $\begin{array}{l}\text { Period 5 } \\
(161-200)\end{array}$ & $\begin{array}{l}\text { Period } 6 \\
(201-240)\end{array}$ & $\begin{array}{l}\text { Period } 7 \\
(241-280)\end{array}$ & $p$-value \\
\hline (b) & & & & & & & & \\
\hline $\begin{array}{l}\text { Age, years, mean } \\
\text { (SD) }\end{array}$ & $60(13)$ & $57(17)$ & $55(18)$ & $55(17)$ & $60(14)$ & $64(11)$ & $66(11)$ & 0.003 \\
\hline $\begin{array}{l}\text { BMI, } \mathrm{kg} / \mathrm{m}^{2} \text {, mean } \\
\text { (SD) }\end{array}$ & $25.5(4.9)$ & $25.7(4.9)$ & $24.9(4.2)$ & $25.3(4.9)$ & $26.3(5.2)$ & $26.7(5.6)$ & $26.3(4.7)$ & 0.66 \\
\hline PUAS, $n(\%)$ & $19(47.5 \%)$ & $16(40 \%)$ & $15(37.5 \%)$ & $20(50 \%)$ & $12(30 \%)$ & $20(50 \%)$ & $15(37.5 \%)$ & 0.46 \\
\hline ASA $\geq \mathrm{III}, n(\%)$ & $14(35 \%)$ & $9(22.5 \%)$ & $8(20 \%)$ & $12(30 \%)$ & $15(37.5 \%)$ & $17(42.5 \%)$ & $10(25 \%)$ & 0.25 \\
\hline $\begin{array}{l}\text { Diagnosis (PDAC), } \\
n(\%)\end{array}$ & $7(17.5 \%)$ & $3(7.5 \%)$ & $4(10 \%)$ & $8(20 \%)$ & $15(37.5 \%)$ & $10(25 \%)$ & $15(37.5 \%)$ & 0.003 \\
\hline $\begin{array}{l}\text { Tumor size, mm, } \\
\text { median (range) }\end{array}$ & $27(4-75)$ & $40(15-110)$ & $33(8-110)$ & $38(10-115)$ & $39(12-130)$ & $29(8-85)$ & $35(11-145)$ & 0.07 \\
\hline $\begin{array}{r}\text { Spleen-preserving } \\
\text { procedure, } n(\%)\end{array}$ & $8(20 \%)$ & $15(37.5 \%)$ & $5(12.5 \%)$ & $13(32.5 \%)$ & $1(2.5 \%)$ & $1(2.5 \%)$ & $2(5 \%)$ & 0.001 \\
\hline $\begin{array}{l}\text { Subtotal distal } \\
\text { pancreatectomy, } \\
n(\%)\end{array}$ & $2(5 \%)$ & $12(30 \%)$ & $5(12.5 \%)$ & $7(17.5 \%)$ & $11(27.5 \%)$ & $6(15 \%)$ & $9(22.5 \%)$ & 0.06 \\
\hline $\begin{array}{l}\text { Multivisceral resec- } \\
\text { tion, } n(\%)\end{array}$ & $5(12.5 \%)$ & $6(15 \%)$ & $4(10 \%)$ & $10(25 \%)$ & $8(20 \%)$ & $3(7.5 \%)$ & $10(25 \%)$ & 0.19 \\
\hline $\begin{array}{l}\text { Blood loss, ml, } \\
\text { median (range) }\end{array}$ & $180(30-3000)$ & $230(10-6250)$ & $50(30-2700)$ & $100(30-2100)$ & $50(30-1600)$ & $200(20-2000)$ & $100(30-3000)$ & 0.23 \\
\hline $\begin{array}{l}\text { Operative time, min, } \\
\text { median (range) }\end{array}$ & $220(123-520)$ & $198(107-480)$ & $158(30-268)$ & $132(56-319)$ & $147(30-386)$ & $143(45-339)$ & $142(62-303)$ & $<0.001$ \\
\hline $\begin{array}{l}\text { Intraoperative } \\
\text { adverse events, } \\
n(\%)\end{array}$ & $7(17.5 \%)$ & $7(17.5 \%)$ & $2(5 \%)$ & $3(7.5 \%)$ & $2(5 \%)$ & $9(22.5 \%)$ & $4(10 \%)$ & 0.1 \\
\hline Conversion, $n(\%)$ & $1(2.5 \%)$ & $2(5 \%)$ & $0(0 \%)$ & $0(0 \%)$ & $0(0 \%)$ & $0(0 \%)$ & $0(0 \%)$ & 0.38 \\
\hline $\begin{array}{l}\text { Postoperative com- } \\
\text { plications, } n(\%)\end{array}$ & $17(42.5 \%)$ & $9(22.5 \%)$ & $10(25 \%)$ & $17(42.5 \%)$ & $16(40 \%)$ & $14(35 \%)$ & $13(32.5 \%)$ & 0.32 \\
\hline $\begin{array}{l}\text { Severe complica- } \\
\text { tions, } n(\%)\end{array}$ & $10(25 \%)$ & $6(15 \%)$ & $6(15 \%)$ & $11(27.5 \%)$ & $9(22.5 \%)$ & $6(15 \%)$ & $7(17.5 \%)$ & 0.64 \\
\hline
\end{tabular}


Table 3 (continued)

\begin{tabular}{|c|c|c|c|c|c|c|c|c|}
\hline Parameters & $\begin{array}{l}\text { Period } 1 \\
(1-40)\end{array}$ & $\begin{array}{l}\text { Period } 2 \\
(41-80)\end{array}$ & $\begin{array}{l}\text { Period } 3 \\
(81-120)\end{array}$ & $\begin{array}{l}\text { Period } 4 \\
(121-160)\end{array}$ & $\begin{array}{l}\text { Period 5 } \\
(161-200)\end{array}$ & $\begin{array}{l}\text { Period } 6 \\
(201-240)\end{array}$ & $\begin{array}{l}\text { Period } 7 \\
(241-280)\end{array}$ & $p$-value \\
\hline \multicolumn{9}{|l|}{ (b) } \\
\hline CR-PF, $n(\%)$ & $5(12.5 \%)$ & $6(15 \%)$ & $2(5 \%)$ & $7(17.5 \%)$ & $11(27.5 \%)$ & $9(22.5 \%)$ & $4(10 \%)$ & 0.11 \\
\hline $\begin{array}{l}\text { Grade } \mathrm{B} / \mathrm{C} \text { hemor- } \\
\text { rhage, } n(\%)\end{array}$ & $3(7.5 \%)$ & $0(0 \%)$ & $2(5 \%)$ & $3(7.5 \%)$ & $3(7.5 \%)$ & $4(10 \%)$ & $2(5 \%)$ & 0.57 \\
\hline Reoperation, $n(\%)$ & $1(2.5 \%)$ & $0(0 \%)$ & $2(5 \%)$ & $3(7.5 \%)$ & $5(12.5 \%)$ & $1(2.5 \%)$ & $4(10 \%)$ & 0.19 \\
\hline Mortality, $n(\%)$ & $0(0 \%)$ & $0(0 \%)$ & $1(2.5 \%)$ & $0(0 \%)$ & $1(2.5 \%)$ & $1(2.5 \%)$ & $0(0 \%)$ & 1.0 \\
\hline $\begin{array}{l}\text { Hospital stay, days, } \\
\text { median (range) }\end{array}$ & $6(2-16)$ & $6(2-30)$ & $5(3-32)$ & $6(2-81)$ & $5(3-34)$ & $5(2-22)$ & $5(3-18)$ & 0.38 \\
\hline
\end{tabular}

\begin{tabular}{|c|c|c|c|c|c|}
\hline $\begin{array}{l}\text { Parameters } \\
\text { (c) }\end{array}$ & $1-10$ & $11-20$ & $21-30$ & $31-40$ & $p$-value \\
\hline Age, years, mean (SD) & $58(12.4)$ & $65.7(6.3)$ & $60(13)$ & $57.6(13.2)$ & 0.39 \\
\hline BMI, $\mathrm{kg} / \mathrm{m}^{2}$, mean (SD) & $25.2(5.5)$ & $24.1(3.1)$ & $25.8(3.5)$ & $25.9(4.3)$ & 0.76 \\
\hline PUAS, $n$ & 3 & 4 & 3 & 5 & 0.89 \\
\hline Diagnosis (PDAC), $n$ & 1 & 0 & 1 & 3 & 0.36 \\
\hline Tumor size, mm, median (range) & $30(8-45)$ & $28(15-55)$ & $28(10-70)$ & $23(7-50)$ & 0.53 \\
\hline Pioneer surgeon assistance/supervision, $n$ & 10 & 3 & 2 & 1 & $<0.01$ \\
\hline Spleen-preserving procedure, $n$ & 4 & 2 & 3 & 6 & 0.38 \\
\hline Multivisceral resection, $n$ & 1 & 0 & 2 & 1 & 0.89 \\
\hline Blood loss, ml, median (range) & $70(30-800)$ & $50(30-400)$ & $75(30-450)$ & $100(30-1200)$ & 0.19 \\
\hline Operative time, min, median (range) & $140(115-205)$ & $193(80-356)$ & $176(110-292)$ & $187(110-284)$ & 0.32 \\
\hline Intraoperative adverse events, $n$ & 1 & 0 & 0 & 2 & 0.6 \\
\hline Conversion, $n$ & 0 & 0 & 0 & 0 & - \\
\hline Postoperative complications, $n$ & 2 & 6 & 6 & 4 & 0.22 \\
\hline Severe complications, $n$ & 2 & 4 & 3 & 3 & 0.96 \\
\hline CR-PF, $n$ & 2 & 3 & 2 & 3 & 1.0 \\
\hline Grade $\mathrm{B} / \mathrm{C}$ hemorrhage, $n$ & 0 & 2 & 2 & 0 & 0.3 \\
\hline Reoperation, $n$ & 0 & 1 & 2 & 0 & 0.6 \\
\hline Mortality, $n$ & 0 & 0 & 0 & 0 & - \\
\hline Hospital stay, days, median (range) & $5(2-13)$ & $8(5-35)$ & $7(2-9)$ & $5(3-11)$ & 0.11 \\
\hline Parameters & $\begin{array}{l}\text { Period } 1 \\
(1-40)\end{array}$ & $\begin{array}{l}\text { Period } 2 \\
(41-80)\end{array}$ & $\begin{array}{l}\text { Period 3 } \\
(81-120)\end{array}$ & $\begin{array}{l}\text { Period } 4 \\
(121-160)\end{array}$ & $p$-value \\
\hline \multicolumn{6}{|l|}{ (d) } \\
\hline Age, years, mean (SD) & $60(12)$ & $63(12)$ & $64(12)$ & $62(14)$ & 0.63 \\
\hline BMI, $\mathrm{kg} / \mathrm{m}^{2}$, mean $(\mathrm{SD})$ & $25.2(4.1)$ & $24.5(3.9)$ & $25.8(4.6)$ & $26.5(4.6)$ & 0.19 \\
\hline PUAS, $n(\%)$ & $15(37.5 \%)$ & $19(47.5 \%)$ & $16(40 \%)$ & $18(45 \%)$ & 0.8 \\
\hline ASA $\geq \mathrm{III}, n(\%)$ & $13(32.5 \%)$ & $10(25 \%)$ & $12(30 \%)$ & $12(30 \%)$ & 0.9 \\
\hline Diagnosis (PDAC), $n(\%)$ & $5(12.5 \%)$ & $12(30 \%)$ & $10(25 \%)$ & $9(22.5 \%)$ & 0.29 \\
\hline Tumor size, mm, median (range) & $28(7-70)$ & $22(7-88)$ & $42(8-127)$ & $40(12-130)$ & 0.002 \\
\hline Pioneer surgeon assistance/supervision, $n$ & $16(40 \%)$ & $1(2.5 \%)$ & $2(5 \%)$ & $0(0 \%)$ & $<0.01$ \\
\hline Spleen-preserving procedure, $n(\%)$ & $15(37.5 \%)$ & $5(12.5 \%)$ & $5(12.5 \%)$ & $6(15 \%)$ & 0.01 \\
\hline Subtotal distal pancreatectomy, $n(\%)$ & $5(12.5 \%)$ & $13(32.5 \%)$ & $10(25 \%)$ & $11(27.5 \%)$ & 0.19 \\
\hline Multivisceral resection, $n(\%)$ & $4(10 \%)$ & $8(20 \%)$ & $6(15 \%)$ & $4(10 \%)$ & 0.51 \\
\hline Blood loss, ml, median (range) & $160(30-1200)$ & $150(30-2500)$ & $180(30-2800)$ & $170(30-1600)$ & 0.27 \\
\hline Operative time, min, median (range) & $175(80-356)$ & $162(92-503)$ & $165(91-428)$ & $166(74-560)$ & 0.78 \\
\hline Intraoperative adverse events, $n(\%)$ & $3(7.5 \%)$ & $6(15 \%)$ & $4(10 \%)$ & $3(7.5 \%)$ & 0.76 \\
\hline Conversion, $n(\%)$ & $0(0 \%)$ & $5(12.5 \%)$ & $1(2.5 \%)$ & $1(2.5 \%)$ & 0.07 \\
\hline Postoperative complications, $n(\%)$ & $18(45 \%)$ & $13(32.5 \%)$ & $14(35 \%)$ & $15(37.5 \%)$ & 0.68 \\
\hline Severe complications, $n(\%)$ & $12(30 \%)$ & $7(17.5 \%)$ & $10(25 \%)$ & $8(20 \%)$ & 0.56 \\
\hline
\end{tabular}


Table 3 (continued)

\begin{tabular}{|c|c|c|c|c|c|}
\hline Parameters & $\begin{array}{l}\text { Period } 1 \\
(1-40)\end{array}$ & $\begin{array}{l}\text { Period } 2 \\
(41-80)\end{array}$ & $\begin{array}{l}\text { Period } 3 \\
(81-120)\end{array}$ & $\begin{array}{l}\text { Period } 4 \\
(121-160)\end{array}$ & $p$-value \\
\hline \multicolumn{6}{|l|}{ (d) } \\
\hline CR-PF, $n(\%)$ & $10(25 \%)$ & $8(20 \%)$ & $5(12.5 \%)$ & $7(17.5 \%)$ & 0.55 \\
\hline Grade $\mathrm{B} / \mathrm{C}$ hemorrhage, $n(\%)$ & $4(10 \%)$ & $2(5 \%)$ & $2(5 \%)$ & $2(5 \%)$ & 0.84 \\
\hline Reoperation, $n(\%)$ & $3(7.5 \%)$ & $1(2.5 \%)$ & $4(10 \%)$ & $3(7.5 \%)$ & 0.69 \\
\hline Mortality, $n(\%)$ & $0(0 \%)$ & $1(2.5 \%)$ & $0(0 \%)$ & $0(0 \%)$ & 1.0 \\
\hline Hospital stay, days, median (range) & $6(2-35)$ & $5(2-31)$ & $5(2-44)$ & $5(3-29)$ & 0.4 \\
\hline
\end{tabular}

$B M I$ body mass index, $P U A S$ previous upper abdominal surgery, $P D A C$ pancreatic ductal adenocarcinoma, $C R-P F$ clinically relevant pancreatic fistula

${ }^{\text {a }}$ Significant difference between the periods 1 and $2-4$

${ }^{\mathrm{b}}$ Significant difference between the periods $1-3$ and 4

only $2 \%$. In contrast, recent data from the national registries and high-volume pancreatic centers report 15-20\% and 15-19\% conversion for MIDP, respectively [25-29]. In half of our patients, conversion was necessary due to the need for major vascular resection and reconstruction, while in the rest, either intraoperative bleeding or suspicion of major vascular involvement was present. This suggests that, in our hands, vascular affection and major intraoperative bleeding were the main predictors of conversion in LDP.

Another important finding of this report was that no significant changes in intra- and postoperative outcomes was observed throughout the first 40 LDPs performed by each surgeon. Furthermore, 9 of 14 conversions reported occurred in surgeons who had already surpassed the experience with the first 40 cases. This is in contrast with the data in the literature suggesting improvement in operative time, estimated blood loss and conversion rate following 10-20 LDPs [9-11, $14,26]$. When analyzing the experience with LDP following the first 40 cases, significant reduction in operative time was demonstrated after 80 cases for the pioneer surgeon. The same trend was not present for the first adopter (expert) although tumor size significantly increased after the first 80 cases without significantly affecting the intra- and postoperative outcomes. The fact that the proportion for PDAC and tumor size increased for the pioneer and expert over time likely led to a decrease in utilization of spleen-preserving LDP. Analysis of the first 40 procedures performed by the adopters demonstrated steady decrease of senior surgeon assistance/supervision without compromising the intraand postoperative outcomes. These findings suggest smooth transition for the adopters as their results did not significantly change when the oversight from senior surgeons was stopped, and more complex cases were undertaken.

Single-surgeon series published by de Rooij and coworkers suggest improvement in Clavien-Dindo grade $\geq$ III complications, grade $\mathrm{B} / \mathrm{C}$ pancreatic fistula and hospital stay following the first 30 cases [12]. These findings were confirmed also in the multicenter study from 11 tertiary referral centers in the UK [15]. In that study, though, the minimally-invasive approach was applied in only half of the patients undergoing distal pancreatectomy. In contrast, there was almost no patient selection in our center as $95 \%$ of distal pancreatectomies were performed laparoscopically. No significant changes in postoperative outcomes were observed for any of the four above mentioned throughout the study period. This is in line with the report from Malleo et al., although they used LDP mainly for benign and low-grade malignancies [30].

Evaluation of the first 30 LDPs performed by the adopters without senior surgeon supervision demonstrated reduced operative time compared to the results of the pioneer surgeon. Superior outcomes for the trainees have also been demonstrated by Nakamura and co-workers [31]. However, one should consider that, in our study, in contrast with the pioneer the adopters had assisted 7-11 LDPs before undertaking their first procedure. Furthermore, slightly less than a half of their first 40 cases were assisted/supervised by a senior surgeon. Finally, less multivisceral and spleen-preserving procedures were performed by the trainees compared to the pioneer and expert indicating patient selection in the early phases of their learning curve.

This study has several strengths and limitations. One of the main advantages that distinguishes this study from similar publications is that it depicts the individual performance of 4 surgeons with different background and experience in a high-volume center. Furthermore, their outcomes with LDP was analyzed in different stages of their learning curves. Another advantage of this report is based on a large material encompassing more than 20 years of experience with this procedure. Most importantly, $95 \%$ of our patients were referred to LDP, which significantly reduces the risk of selection bias. The main drawback remains the retrospective observational design of this study with all inherent biases. Nearly $13 \%$ of the patients $(n=80)$ were 
Table 4 The initial experience of the trainees with laparoscopic distal pancreatectomy: trainee 1 (a) and trainee 2 (b)

\begin{tabular}{|c|c|c|c|c|c|}
\hline $\begin{array}{l}\text { Parameters } \\
\text { (a) }\end{array}$ & $1-10$ & $11-20$ & $21-30$ & $31-39$ & $p$-value \\
\hline Age, years, mean (SD) & $62.8(12.3)$ & $61.7(13.7)$ & $59(15.5)$ & $57.4(14.3)$ & 0.83 \\
\hline BMI, $\mathrm{kg} / \mathrm{m}^{2}$, mean $(\mathrm{SD})$ & $25.2(3)$ & $28.7(6.1)$ & $27.4(5.3)$ & $28.3(6)$ & 0.45 \\
\hline PUAS, $n$ & 3 & 5 & 4 & 5 & 0.77 \\
\hline Diagnosis (PDAC), $n$ & 0 & 1 & 1 & 2 & 0.43 \\
\hline Tumor size, mm, median (range) & $47(11-114)$ & $47.5(20-95)$ & $35(16-60)$ & $50(27-80)$ & 0.37 \\
\hline Pioneer/expert surgeon assistance/supervision, $n$ & 5 & 6 & 3 & 0 & 0.025 \\
\hline Spleen-preserving procedure, $n$ & 0 & 1 & 0 & 0 & 1.0 \\
\hline Multivisceral resection, $n$ & 0 & 1 & 1 & 1 & 0.89 \\
\hline Blood loss, ml, median (range) & $50(30-300)$ & $125(50-3700)$ & $50(20-250)$ & $100(10-400)$ & 0.27 \\
\hline Operative time, min, median (range) & $149(100-202)$ & $162(125-341)$ & $159(78-314)$ & $188(122-219)$ & 0.36 \\
\hline Intraoperative adverse events, $n$ & 0 & 3 & 0 & 2 & 0.08 \\
\hline Conversion, $n$ & 0 & 1 & 0 & 0 & 1.0 \\
\hline Postoperative complications, $n$ & 5 & 3 & 3 & 1 & 0.4 \\
\hline Severe complications, $n$ & 4 & 2 & 2 & 1 & 0.56 \\
\hline CR-PF, $n$ & 2 & 2 & 2 & 1 & 1.0 \\
\hline Grade $\mathrm{B} / \mathrm{C}$ hemorrhage, $n$ & 1 & 1 & 1 & 0 & 1.0 \\
\hline Reoperation, $n$ & 0 & 1 & 1 & 0 & 1.0 \\
\hline Mortality, $n$ & 0 & 0 & 0 & 0 & - \\
\hline Hospital stay, days, median (range) & $7(4-19)$ & $4(3-38)$ & $6(3-13)$ & $4(3-7)$ & 0.08 \\
\hline $\begin{array}{l}\text { Parameters } \\
\text { (b) }\end{array}$ & $1-10$ & $11-20$ & $21-30$ & $31-40$ & $p$-value \\
\hline Age, years, mean (SD) & $68.3(6.8)$ & $60(16.3)$ & $66.4(8.1)$ & $67.2(11.1)$ & 0.36 \\
\hline BMI, $\mathrm{kg} / \mathrm{m}^{2}$, mean (SD) & $26.5(4.8)$ & $26.9(2.9)$ & $27.3(3.3)$ & $28(4.3)$ & 0.85 \\
\hline PUAS, $n$ & 4 & 5 & 5 & 7 & 0.68 \\
\hline Diagnosis (PDAC), $n$ & 1 & 3 & 4 & 3 & 0.6 \\
\hline Tumor size, mm, median (range) & $22(14-37)$ & $25(9-100)$ & $35(13-80)$ & $30(11-110)$ & 0.38 \\
\hline Pioneer/expert surgeon assistance/supervision, $n$ & 7 & 4 & 5 & 0 & 0.008 \\
\hline Spleen-preserving procedure, $n$ & 1 & 0 & 1 & 0 & 1.0 \\
\hline Multivisceral resection, $n$ & 0 & 0 & 1 & 0 & 1.0 \\
\hline Blood loss, ml, median (range) & $50(30-500)$ & $50(30-800)$ & $50(30-200)$ & $50(20-500)$ & 0.88 \\
\hline Operative time, min, median (range) & $165(95-267)$ & $186(148-325)$ & $156(71-389)$ & $139(76-341)$ & 0.07 \\
\hline Intraoperative adverse events, $n$ & 0 & 2 & 0 & 1 & 0.6 \\
\hline Conversion, $n$ & 0 & 1 & 0 & 1 & 1.0 \\
\hline Postoperative complications, $n$ & 1 & 3 & 2 & 4 & 0.62 \\
\hline Severe complications, $n$ & 0 & 3 & 0 & 2 & 0.14 \\
\hline CR-PF, $n$ & 0 & 3 & 0 & 2 & 0.14 \\
\hline Grade $\mathrm{B} / \mathrm{C}$ hemorrhage, $n$ & 0 & 0 & 0 & 0 & - \\
\hline Reoperation, $n$ & 0 & 0 & 0 & 1 & 1.0 \\
\hline Mortality, $n$ & 0 & 0 & 0 & 0 & - \\
\hline Hospital stay, days, median (range) & $4(2-5)$ & $5(3-40)$ & $5(3-6)$ & $5(3-9)$ & 0.19 \\
\hline
\end{tabular}

$B M I$ body mass index, $P U A S$ previous upper abdominal surgery, $P D A C$ pancreatic ductal adenocarcinoma, $C R-P F$ clinically relevant pancreatic fistula

not analyzed as they were operated by other surgeons at the department. At the same time, the majority $(64 \%)$ of those procedures were assisted and supervised by the pioneer or expert. Furthermore, the postoperative outcomes of these 80 cases were not significantly different from those demonstrated by surgeons included in this study (data not shown).

Our findings suggest that standardization LDP technique and stepwise training in a high-volume center can significantly reduce the conversion rate providing satisfactory results for 
Table 5 Experiences with the first 30 laparoscopic distal pancreatectomies for the pioneer and the adopters without senior surgeon assistance

\begin{tabular}{|c|c|c|c|c|c|}
\hline Parameters & Pioneer & Expert & Trainee 1 & Trainee 2 & $p$-value \\
\hline Within individual LDP series & $1-30$ & $1-46$ & $1-39$ & $1-48$ & \\
\hline Age, years, mean (SD) & $58.6(13.7)$ & $63.6(10.7)$ & $60.1(13.3)$ & $63.4(12.1)$ & 0.33 \\
\hline BMI, $\mathrm{kg} / \mathrm{m}^{2}$, mean (SD) & $25.9(5.4)$ & $24.8(3.3)$ & $27.4(4.8)$ & $26.5(3.9)$ & 0.13 \\
\hline $\mathrm{ASA} \geq \mathrm{III}, n(\%)$ & $10(33.3 \%)$ & $11(36.7 \%)$ & $10(33.3 \%)$ & $13(43.3 \%)$ & 0.72 \\
\hline PUAS, $n(\%)$ & $16(53.3 \%)$ & $14(46.7 \%)$ & $14(46.7 \%)$ & $15(50 \%)$ & 0.95 \\
\hline Diagnosis (PDAC), $n(\%)$ & $5(16.7 \%)$ & $7(23.3 \%)$ & $3(10 \%)$ & $8(26.7 \%)$ & 0.37 \\
\hline Tumor size, mm, median (range) & $25(4-80)$ & $25(7-88)$ & $37(13-80)$ & $31(11-110)$ & 0.15 \\
\hline Spleen-preserving procedure, $n(\%)$ & $7(23.3 \%)$ & $11(36.7 \%)$ & $1(3.3 \%)$ & $1(3.3 \%)$ & $0.001^{+}$ \\
\hline Multivisceral resection, $n(\%)$ & $4(13.3 \%)$ & $6(20 \%)$ & $1(3.3 \%)$ & $0(0 \%)$ & $\mathbf{0 . 0 2} \neq$ \\
\hline Blood loss, ml, median (range) & $120(30-1500)$ & $50(30-900)$ & $80(10-400)$ & $50(20-750)$ & 0.2 \\
\hline Operative time, min, median (range) & $230(123-520)$ & $188(110-356)$ & $161(78-314)$ & $156(71-341)$ & $<0.001 *$ \\
\hline Intraoperative adverse events, $n(\%)$ & $5(16.7 \%)$ & $3(10 \%)$ & $3(10 \%)$ & $3(10 \%)$ & 0.89 \\
\hline Conversion, $n(\%)$ & $1(3.3 \%)$ & $2(6.7 \%)$ & $0(0 \%)$ & $1(3.3 \%)$ & 0.9 \\
\hline Postoperative complications, $n(\%)$ & $13(43.3 \%)$ & $11(36.7 \%)$ & $8(26.7 \%)$ & $9(30 \%)$ & 0.54 \\
\hline Severe complications, $n(\%)$ & $8(26.7 \%)$ & $7(23.3 \%)$ & $7(23.3 \%)$ & $5(16.7 \%)$ & 0.87 \\
\hline CR-PF, $n(\%)$ & $4(13.3 \%)$ & $5(16.7 \%)$ & $4(13.3 \%)$ & $4(13.3 \%)$ & 1.0 \\
\hline Grade $\mathrm{B} / \mathrm{C}$ hemorrhage, $n(\%)$ & $2(6.7 \%)$ & $3(10 \%)$ & $2(6.7 \%)$ & $1(3.3 \%)$ & 0.96 \\
\hline Reoperation, $n(\%)$ & $0(0 \%)$ & $2(6.7 \%)$ & $1(3.3 \%)$ & $1(3.3 \%)$ & 0.9 \\
\hline Mortality, $n(\%)$ & $0(0 \%)$ & $0(0 \%)$ & $0(0 \%)$ & $0(0 \%)$ & - \\
\hline Readmission, $n(\%)$ & $3(10 \%)$ & $3(10 \%)$ & $4(13.3 \%)$ & $4(13.3 \%)$ & 1.0 \\
\hline Hospital stay, days, median (range) & $6(2-12)$ & $6(2-35)$ & $5(3-19)$ & $4(2-40)$ & $0.02^{\text {II }}$ \\
\hline
\end{tabular}

$B M I$ body mass index, $P U A S$ previous upper abdominal surgery, $P D A C$ pancreatic ductal adenocarcinoma, $C R-P F$ clinically relevant pancreatic fistula

${ }^{+}$Significant difference between the pioneer/expert and trainees

F Significant difference between the expert and trainees

*Significant difference between the pioneer and others

"I Significant difference between the pioneer/expert and trainee 2

different surgeons. No significant improvement in perioperative outcomes of LDP has been detected throughout the first 40 LDPs of each surgeon. Furthermore, 80 procedures seem to be more realistic in the setting when almost no patient selection is present. The exact number of procedures required to overcome the learning curve is difficult to determine as it seems to depend on patient selection policy and surgical training program at the corresponding center.

Supplementary Information The online version of this article (https:// doi.org/10.1007/s00464-021-08306-3) contains supplementary material, which is available to authorized users.

Funding Open Access funding provided by University of Oslo (incl Oslo University Hospital).

\section{Compliance with ethical standards}

Disclosures All the authors, Dr. Sahakyan, Dr. Røsok, Dr. Tholfsen, Dr. Kleive, Dr. Waage, Dr. Ignjatovic, Dr. Buanes, Dr. Labori and Dr. Edwin have no conflicts of interest or financial ties to disclose.

Open Access This article is licensed under a Creative Commons Attribution 4.0 International License, which permits use, sharing, adaptation, distribution and reproduction in any medium or format, as long as you give appropriate credit to the original author(s) and the source, provide a link to the Creative Commons licence, and indicate if changes were made. The images or other third party material in this article are included in the article's Creative Commons licence, unless indicated otherwise in a credit line to the material. If material is not included in the article's Creative Commons licence and your intended use is not permitted by statutory regulation or exceeds the permitted use, you will need to obtain permission directly from the copyright holder. To view a copy of this licence, visit http://creativecommons.org/licenses/by/4.0/. 


\section{References}

1. Vollmer CM, Asbun HJ, Barkun J, Besselink MG, Boggi U, Conlon KC, Han HS, Hansen PD, Kendrick ML, Montagnini AL, Palanivelu C, Røsok BI, Shrikhande SV, Wakabayashi G, Zeh HJ, Kooby DA (2017) Proceedings of the first international stateof-the-art conference on minimally-invasive pancreatic resection (MIPR). HPB (Oxford) 19(3):171-177

2. Asbun HJ, Moekotte AL, Vissers FL, Kunzler F, Cipriani F, Alseidi A, D’Angelica MI, Balduzzi A, Bassi C, Björnsson B, Boggi U, Callery MP, Del Chiaro M, Coimbra FJ, Conrad C, Cook A, Coppola A, Dervenis C, Dokmak S, Edil BH, Edwin B, Giulianotti PC, Han HS, Hansen PD, van der Heijde N, van Hilst J, Hester CA, Hogg ME, Jarufe N, Jeyarajah DR, Keck T, Kim SC, Khatkov IE, Kokudo N, Kooby DA, Korrel M, de Leon FJ, Lluis N, Lof S, Machado MA, Demartines N, Martinie JB, Merchant NP, Molenaar QI, Moravek C, Mou YP, Nakamura M, Nealon WH, Palanivelu C, Pessaux P, Pitt HA, Polanco PM, Primrose JN, Rawashdeh A, Sanford DE, Senthilnathan P, Shrikhande SV, Stauffer JA, Takaori K, Talamonti MS, Tang CN, Vollmer CM, Wakabayashi G, Walsh RM, Wang SE, Zinner MJ, Wolfgang CL, Zureikat AH, Zwart MJ, Conlon KC, Kendrick ML, Zeh HJ, Abu Hilal M, Besselink MG (2020) The Miami international evidencebased guidelines on minimally invasive pancreas resection. Ann Surg 271(1):1-14

3. de Rooij T, Besselink MG, Shamali A, Butturini G, Busch OR, Edwin B, Troisi R, Fernández-Cruz L, Dagher I, Bassi C, Abu Hilal M, DIPLOMA Trial Group (2016) Pan-European survey on the implementation of minimally invasive pancreatic surgery with emphasis on cancer. HPB (Oxford) 18(2):170-176

4. van Hilst J, de Rooij T, Abu Hilal M, Asbun HJ, Barkun J, Boggi U, Busch OR, Conlon KCP, Dijkgraaf MG, Han HS, Hansen PD, Kendrick ML, Montagnini AL, Palanivelu C, Røsok BI, Shrikhande SV, Wakabayashi G, Zeh HJ, Vollmer CM, Kooby DA, Besselink MGH (2017) Worldwide survey on opinions and use of minimally invasive pancreatic resection. HPB (Oxford) 19(3):190-204

5. de Rooij T, van Hilst J, van Santvoort H, Boerma D, van den Boezem P, Daams F, van Dam R, Dejong C, van Duyn E, Dijkgraaf M, van Eijck C, Festen S, Gerhards M, Groot Koerkamp B, de Hingh I, Kazemier G, Klaase J, de Kleine R, van Laarhoven C, Luyer M, Patijn G, Steenvoorde P, Suker M, Abu Hilal M, Busch O, Besselink M; Dutch Pancreatic Cancer Group (2019) Minimally invasive versus open distal pancreatectomy (LEOPARD): a multicenter patient-blinded randomized controlled trial. Ann Surg 269(1):2-9

6. Bjornsson B, Larsson AL, Hjalmarsson C, Gasslander T, Sandstrom P (2020) Comparison of the duration of hospital stay after laparoscopic or open distal pancreatectomy: randomized controlled trial. Br J Surg. https://doi.org/10.1002/bjs.11554

7. Edwin B, Sahakyan MA, Hilal MA, Besselink MG, Braga M, Fabre JM, Fernández-Cruz L, Gayet B, Kim SC, Khatkov IE, EAES Consensus Conference Study Group (2017) Laparoscopic surgery for pancreatic neoplasms: the European association for endoscopic surgery clinical consensus conference. Surg Endosc 31(5):2023-2041

8. Palanivelu C, Takaori K, Abu Hilal M, Kooby DA, Wakabayashi G, Agarwal A, Berti S, Besselink MG, Chen KH, Gumbs AA, Han HS, Honda G, Khatkov I, Kim HJ, Li JT, Long TCD, Machado MA, Matsushita A, Menon K, Min-Hua Z, Nakamura M, Nagakawa Y, Pekolj J, Poves I, Rahman S, Rong L, Sa Cunha A, Senthilnathan P, Shrikhande SV, Gurumurthy SS, Yoon DS, Yoon YS, Khatri VP (2018) International Summit on Laparoscopic Pancreatic Resection (ISLPR) "Coimbatore Summit Statements." Surg Oncol 27(1):A10-A15
9. Barrie J, Ammori BJ (2015) Minimally invasive distal pancreatectomy: a single-center analysis of outcome with experience and systematic review of the literature. Surg Laparosc Endosc Percu$\tan$ Tech 25(4):297-302

10. Ricci C, Casadei R, Buscemi S, Taffurelli G, D’Ambra M, Pacilio CA, Minni F (2015) Laparoscopic distal pancreatectomy: what factors are related to the learning curve? Surg Today 45(1):50-56

11. Braga M, Ridolfi C, Balzano G, Castoldi R, Pecorelli N, Di Carlo V (2012) Learning curve for laparoscopic distal pancreatectomy in a high-volume hospital. Updates Surg 64(3):179-183

12. de Rooij T, Cipriani F, Rawashdeh M, van Dieren S, Barbaro S, Abuawwad M, van Hilst J, Fontana M, Besselink MG, Abu Hilal M (2017) Single-surgeon learning curve in 111 laparoscopic distal pancreatectomies: does operative time tell the whole story? J Am Coll Surg 224(5):826-832.e1

13. Kim HS, Park JS, Yoon DS (2019) True learning curve of laparoscopic spleen-preserving distal pancreatectomy with splenic vessel preservation. Surg Endosc 33(1):88-93

14. Nachmany I, Pencovich N, Ben-Yehuda A, Lahat G, Nakache R, Goykhman Y, Lubezky N, Klausner JM (2016) Laparoscopic distal pancreatectomy: learning curve and experience in a tertiary center. J Laparoendosc Adv Surg Tech A 26(6):470-474

15. Lof S, Moekotte AL, Al-Sarireh B, Ammori B, Aroori S, Durkin D, Fusai GK, French JJ, Gomez D, Marangoni G, Marudanayagam R, Soonawalla Z, Sutcliffe R, White SA, Abu Hilal M, Minimally Invasive Liver and Pancreatic Surgery Study Group - UK (MI-LAPS UK) (2019) Multicentre observational cohort study of implementation and outcomes of laparoscopic distal pancreatectomy. Br J Surg 106(12):1657-1665

16. Hartwig W, Vollmer CM, Fingerhut A, Yeo CJ, Neoptolemos JP, Adham M, Andrén-Sandberg A, Asbun HJ, Bassi C, Bockhorn M, Charnley R, Conlon KC, Dervenis C, Fernandez-Cruz L, Friess H, Gouma DJ, Imrie CW, Lillemoe KD, Milićević MN, Montorsi M, Shrikhande SV, Vashist YK, Izbicki JR, Büchler MW, International Study Group on Pancreatic Surgery (2014) Extended pancreatectomy in pancreatic ductal adenocarcinoma: definition and consensus of the International Study Group for Pancreatic Surgery (ISGPS). Surgery 156(1):1-14

17. Kazaryan AM, Rosok BI, Edwin B (2013) Morbidity assessment in surgery: refinement proposal based on a concept of perioperative adverse events. ISRN Surg 2013:625093

18. Satava RM (2005) Identification and reduction of surgical error using simulation. Minim Invasive Ther Allied Technol 14(4):257-261

19. Strasberg SM, Linehan DC, Hawkins WG (2009) The accordion severity grading system of surgical complications. Ann Surg 250(2):177-186

20. Bassi C, Marchegiani G, Dervenis C, Sarr M, Abu Hilal M, Adam M, Allen P, Andersson R, Asbun HJ, Besselink MG, Conlon K, Del Chiaro M, Falconi M, Fernandez-Cruz L, Fernandez-Del Castillo C, Fingerhut A, Friess H, Gouma DJ, Hackert T, Izbicki J, Lillemoe KD, Neoptolemos JP, Olah A, Schulick R, Shrikhande SV, Takada T, Takaori K, Traverso W, Vollmer CR, Wolfgang CL, Yeo CJ, Salvia R, Buchler M, International Study Group on Pancreatic Surgery (ISGPS) (2016) The 2016 update of the International Study Group (ISGPS) definition and grading of postoperative pancreatic fistula: 11 Years After. Surgery 161(3):584-591

21. Wente MN, Veit JA, Bassi C, Dervenis C, Fingerhut A, Gouma DJ, Izbicki JR, Neoptolemos JP, Padbury RT, Sarr MG, Yeo CJ, Büchler MW (2007) Postpancreatectomy hemorrhage (PPH): an International Study Group of Pancreatic Surgery (ISGPS) definition. Surgery 142(1):20-25

22. Mise Y, Day RW, Vauthey JN, Brudvik KW, Schwarz L, Prakash L, Parker NH, Katz MHG, Conrad C, Lee JE, Fleming JB, Aloia TA (2016) After pancreatectomy, the "90 Days from Surgery" definition is superior to the "30 Days from Discharge" definition 
for capture of clinically relevant readmissions. J Gastrointest Surg 20(1):77-84

23. Edwin B, Mala T, Mathisen O, Gladhaug I, Buanes T, Lunde OC, Søreide O, Bergan A, Fosse E (2004) Laparoscopic resection of the pancreas: a feasibility study of the short-term outcome. Surg Endosc 18(3):407-411

24. Rosok BI, Marangos IP, Kazaryan AM, Rosseland AR, Buanes T, Mathisen O, Edwin B (2010) Single-centre experience of laparoscopic pancreatic surgery. Br J Surg 97(6):902-909

25. Casadei R, Ricci C, Pacilio CA, Ingaldi C, Taffurelli G, Minni F (2018) Laparoscopic distal pancreatectomy: which factors are related to open conversion? Lessons learned from 68 consecutive procedures in a high-volume pancreatic center. Surg Endosc 32(9):3839-3845

26. Hua Y, Javed AA, Burkhart RA, Makary MA, Weiss MJ, Wolfgang CL, He J (2017) Preoperative risk factors for conversion and learning curve of minimally invasive distal pancreatectomy. Surgery 162(5):1040-1047

27. Beane JD, Pitt HA, Dolejs SC, Hogg ME, Zeh HJ, Zureikat AH (2018) Assessing the impact of conversion on outcomes of minimally invasive distal pancreatectomy and pancreatoduodenectomy. HPB (Oxford) 20(4):356-363

28. Nassour I, Wang SC, Porembka MR, Augustine MM, Yopp AC, Mansour JC, Minter RM, Choti MA, Polanco PM (2017)
Conversion of minimally invasive distal pancreatectomy: predictors and outcomes. Ann Surg Oncol 24(12):3725-3731

29. Zureikat AH, Borrebach J, Pitt HA, McGill D, Hogg ME, Thompson V, Bentrem DJ, Hall BL, Zeh HJ (2017) Minimally invasive hepatopancreatobiliary surgery in North America: an ACS-NSQIP analysis of predictors of conversion for laparoscopic and robotic pancreatectomy and hepatectomy. HPB (Oxford) 19(7):595-602

30. Malleo G, Damoli I, Marchegiani G, Esposito A, Marchese T, Salvia $R$, Bassi C, Butturini G (2015) Laparoscopic distal pancreatectomy: analysis of trends in surgical techniques, patient selection, and outcomes. Surg Endosc 29(7):1952-1962

31. Nakamura Y, Matsushita A, Katsuno A, Sumiyoshi H, Yoshioka M, Shimizu T, Mizuguchi Y, Uchida E (2014) Laparoscopic distal pancreatectomy: educating surgeons about advanced laparoscopic surgery. Asian J Endosc Surg 7(4):295-300

Publisher's Note Springer Nature remains neutral with regard to jurisdictional claims in published maps and institutional affiliations. 